Research Paper

\title{
Pulsed radiofrequency attenuates diabetic neuropathic pain and suppresses formalin-evoked spinal glutamate release in rats
}

\author{
Yu-Hsin Huang ${ }^{1 *}$, Shao-Yun Hou1 ${ }^{*}$, Jen-Kun Cheng ${ }^{2}$, Chih-Hsien Wu ${ }^{1}$, Chung-Ren Lin ${ }^{1} \bowtie$ \\ 1. Department of Anesthesiology, Chang Gung Memorial Hospital-Kaohsiung Medical Centre, Chang Gung University College of Medicine, Kaohsiung, \\ Taiwan. \\ 2. Department of Anesthesiology, MacKay Memorial Hospital, Taipei, Taiwan. \\ *Y. H. Huang and S. Y. Sou contributed equally to this project and should be considered co-first authors. \\ $\square$ Corresponding author: 123 Da-Pei Rd, Niao-Sung District, Kaohsiug, 833, Taiwan. TEL: +88677317123 ext. 2788 E-mail: chungren@ntu.edu.tw. \\ (c) Ivyspring International Publisher. This is an open access article distributed under the terms of the Creative Commons Attribution (CC BY-NC) license \\ (https:// creativecommons.org/licenses/by-nc/4.0/). See http://ivyspring.com/terms for full terms and conditions.
}

Received: 2016.05.05; Accepted: 2016.11.01; Published: 2016.12.08

\begin{abstract}
BACKGROUND: Pulsed radiofrequency (PRF) has been used to treat chronic pain for years, but its effectiveness and mechanism in treating diabetic neuropathic pain are still unexplored. The aim of this study was to elucidate the modulation of diabetic neuropathic pain induced by streptozotocin and the release of spinal excitatory amino acids by PRF.

METHODS: Diabetes was induced by intraperitoneal administration of streptozotocin. Pulsed radiofrequency was applied to $L 5$ and $L 6$ dorsal roots at $42^{\circ} \mathrm{C}$ for $2 \mathrm{~min}$. The responses of all of the groups to thermal, mechanical and cold stimuli were measured for a period of $6 \mathrm{~d}$ after this process. Seven days after PRF treatment, intrathecal microdialysis was used to examine the effect of pulsed radiofrequency on the formalin-evoked spinal release of excitatory amino acids and concurrent behaviour responses from diabetic rats.

RESULTS: Three weeks after intraperitoneal streptozotocin treatment and before PRF application, mechanical, thermal and cold hypersensitivity occurred. Application of PRF significantly alleviated hyperglycaemia-induced mechanical, thermal and cold hypersensitivity and also attenuated the increase in formalin-evoked CSF glutamate concentration, compared with sham treated diabetic rats.

CONCLUSION: It may be concluded that PRF has an analgesic effect on neuropathic pain by suppressing the nociception-induced release of excitatory neurotransmitters. PRF may provide a novel promising therapeutic approach for managing diabetic neuropathic pain.
\end{abstract}

Key words: diabetes, neuropathic pain, pulsed radiofrequency, glutamate, microdialysis.

\section{Introduction}

Diabetic neuropathic pain (DNP) is one of the most challenging complications of diabetes mellitus [1]. Patients with DNP experience distal extremity burning or "shock-like sensations" with increased sensitivity to both painful (hyperalgesia) and non-painful stimuli (allodynia), which can be disabling and devastating $[2,3]$. Despite the high morbidity of DNP, the treatment remains unsatisfied. Current therapeutic options are limited to symptomatic treatment with tricyclic antidepressants, selective serotonin noradrenaline reuptake inhibitors, capsaicin, anticonvulsants, opiates and membrane stabilizers [2, 3]. However, their use is often associated with problems such as insufficient efficacy, development of adverse side effects, and cost effectiveness. The development of better treatment modalities for diabetic neuropathic pain should be based on pathogenetic mechanisms. 
The excitatory amino acids such as glutamate and aspartate are major neurotransmitters released from central and peripheral terminals of nociceptive primary afferent fibres critically important in modulating the transmission of peripheral painful stimuli within the spinal cord [4]. Activation of glutamate receptors underlies central hypersensitivity, whereby the level of transmission of noxious messages is potentiated [5]. The development of diabetic neuropathic pain is associated with increased nociceptive input, neuronal hyperactivity and sustained stimulation of particular glutamate receptors in the spinal cord [6-8].

Pulsed radiofrequency (PRF) has been suggested as a therapeutic modality of various painful conditions, such as radicular pain, facet joint pain, sacroiliac joint pain, and other chronic pain syndromes $[9,10]$. Its popularity has increased significantly in recent years most likely due to its lack of neurodestructive effects and its potential clinical efficacy compared to continuous radiofrequency therapy [11]. PRF offers broader applicability to pathological conditions such as neuropathic pain in which continuous radiofrequency therapy is relatively contraindicated [12].

In this study, we investigated the potential beneficial effect of PRF on hyperalgesia and allodynia in diabetic rats, and on the involvement of EAA release, to examine the mechanism of action of PRF in a DNP pain model.

\section{Materials and Methods}

All experimental protocols were reviewed and approved by the Institutional Animal Care and Use Committee of Chang Gung Memorial Hospital, Taiwan. Adult male Sprague-Dawley rats (250-300 g) were used in this study.

The DNP rat model was produced by intraperitoneally (i.p.) injecting streptozotocin (STZ, $50 \mathrm{mg} / \mathrm{kg}$ dissolved in $5 \mathrm{ml}$ saline) dissolved in saline. The control group received normal saline. Diabetes was confirmed 6 days and 12 days post-injection (glucose $>12 \mathrm{mmol} / \mathrm{L}$ ). Only rats with confirmed diabetes were included. Animals were divided randomly into five groups and treated as follows. Group A (naïve control, $n=10$ ) included naive animals. The rats in group B (NS+sham, $n=10)$ were administered a saline intraperitoneal injection $(5 \mathrm{ml})$ and received PRF electrodes placement without radiofrequency current delivered. The rats in group $\mathrm{C}$ (NS+PRF, $n=10$ ) were administered a saline intraperitoneal injection $(5 \mathrm{ml})$ and received PRF (20 ms of $500 \mathrm{kHz}$ RF pulses, delivered at a rate of $2 \mathrm{~Hz}$; maximum temperature $42{ }^{\circ} \mathrm{C}$ ) to the L5 and L6 anterior primary ramus, just distal to the intervertebral foramen. The rats in group $\mathrm{D}$ (STZ+sham, $n=10$ ) were administered a STZ intraperitoneal injection and received PRF needles placement without radiofrequency current delivered. The rats in group E (STZ+PRF, $n=10)$ were administered a STZ intraperitoneal injection and received PRF to the L5 and L6 anterior primary ramus just distal to the intervertebral foramen. On the $18^{\text {th }}$ day after STZ or saline, all of the animals were implanted with a microdialysis catheter as previously described [13].

In the PRF groups, percutaneous electrode placement was performed as described by Ferrai et al. [14] with some modification. The electrode (10 cm-long, 22 G, $5 \mathrm{~mm}$ active tip; SMK-10; Radionics, Burlington, Massachusetts, USA) was inserted $1.5 \mathrm{~cm}$ laterally to the vertebral column, approximately 0.5 $\mathrm{cm}$ caudal from a line passing the rostral borders of the iliac crests. The electrode was then connected to a PRF generator (RF-5; Radionics) to expose the nerve to RF fields. Proximity of the needle to the nerve was determined by appropriate motor stimulation at $2 \mathrm{~Hz}$ $(0.5 \mathrm{~V})$ was used. The generator output $(500 \mathrm{kHz}$ electric current at two bursts per second of $20 \mathrm{~ms}$ duration each) was increased automatically until an electrode tip temperature of $42{ }^{\circ} \mathrm{C}$ and maintained for $120 \mathrm{~s}$. In the sham-treated rats, identical electrode placement was performed, but no PRF electric current was applied.

A behavioural assessment of thermal and mechanical sensitivity was performed at five time points before and after PRF treatment $(0,3 \mathrm{~h}, 1 \mathrm{~d}, 3 \mathrm{~d}$ and $6 \mathrm{~d}$ after PRF). Immediately after behavioural assessments on the $6^{\text {th }}$ day, body weight and blood glucose in the blood were measured.

To assess the mechanical hypersensitivity, an electrical version of the Von Frey test (Dynamic Plantar Aesthesiometer; Ugo Basile, Milan, Italy) was used. Rats were placed in a Plexiglas chamber, and a servo-controlled mechanical stimulus (a metallic filament of $0.5-\mathrm{mm}$ diameter) was applied to the plantar surface repeatedly at 5-min intervals; the wire exerted a progressively increasing punctuate pressure $(2.5 \mathrm{~g} / \mathrm{s})$ until the rat withdrew the paw. A maximum cut-off value of $50 \mathrm{~g}$ was established to prevent tissue damage. The threshold was determined three times for each time point, and the mean of three experiments was used in the analysis. The MPWT were measured before saline or STZ injection every week for three weeks and then at $3 \mathrm{~h}, 1 \mathrm{~d}, 3 \mathrm{~d}$ and $6 \mathrm{~d}$ after PRF treatment. The experimenter was blinded to treatment classification throughout the study, although body weight loss related to STZ injection was evident during behavioural testing.

To assess the thermal hyperalgesia, a paw 
thermal stimulation system was used to measure the nociceptive response to heat [13]. A radiant heat source was used to stimulate the plantar side of the hind paw. Then, an automatic timer was used to record the paw withdrawal latency in seconds when the rats withdrew the paw. If the animals did not withdraw the paw for $20 \mathrm{~s}$, the heat stimulation was stopped to prevent thermal injury. All of the tests were repeated three times at intervals of $5 \mathrm{~min}$ between each application.

Before the formal experiment of cold allodynia, a series of temperatures was tested in the preliminary studies. It was found that the diabetic rats were sensitive to cold simulation, and $21^{\circ} \mathrm{C}$ was the threshold which elicited significantly different response latency in our study. To minimize the risk of tissue injury by low temperature, the temperature of $21^{\circ} \mathrm{C}$ was used in the present study. Briefly, to apply cold stimulation, rats were placed on a cold stainless steel plate (model 35100, Ugo Basile) maintained at $21^{\circ} \mathrm{C}$. The latency (s) to the first lifting or shaking of the hind paws was measured. If the animals did not withdraw the paw for $180 \mathrm{~s}$, the cold stimulation would be stopped to prevent tissue injury. The cold stimulation was repeated twice at intervals of $5 \mathrm{~min}$ between each application.

\section{Formalin test}

Following 50 microliters of formalin 5\% injection to the left hind paw, the number of flinches of the afflicted paw was counted in 1-min bins for $60 \mathrm{~min}$ post-injection [15]. Phase 1 was quantified as the sum of flinches measured during minutes $0-5$ after paw formalin injection, phase $Q$ as the sum of flinches during minutes 5-6, 10-11 and 15-16 and phase 2 as the sum of flinches during minutes 20-21, 25-26, $30-31,35-36,40-41,45-46,50-51,55-56$ and 60-61.

\section{Intrathecal microdialysis}

The recovery rate of the microdialysis for each catheter was measured before each experiment [13]. After $2 \mathrm{~d}$, the dialysis catheters were perfused with artificial cerebrospinal fluid at a rate of $5 \mu \mathrm{l} / \mathrm{min}$. All experimental manipulations were preceded by a 30-min washout period, followed by two control sample collections (10 min each). Ten microliters of dialysate was assayed using on-line high-performance liquid chromatography (HP1100; Hewlett-Packard, Palo Alto, California, USA) coupled with a fluorescence detector. The detection sensitivity was up to $10^{-8} \mathrm{M}$. Sensitivity was $5-10 \mathrm{pmol} / 10 \mu \mathrm{l}$ tube.

\section{Statistical analysis}

All data in the study were expressed as the mean \pm standard deviation (SD). One way or two-way analysis of variance (ANOVA) was applied to compare the data using SPSS16.0 software (SPSS Inc., Chicago, IL, USA). Tukey's test for single and multiple comparisons, respectively. $\mathrm{P}$ values $<0.05$ were considered statistically significant.

\section{Results}

\section{Metabolic effect of STZ injection in rats}

STZ-injected rats were hyperglycaemic $(18.3 \pm 1.8$ vs. $6.1 \pm 0.9 \mathrm{mmol} / \mathrm{l})$ and lighter $(219 \pm 19.4$ vs. $280.5 \pm 21.2 \mathrm{~g}$ ) than age-matched controls (both $P<0.05)$ at the conclusion of the study.

\section{Effect of PRF on mechanical hypersensitivity}

STZ induced significant mechanical allodynia by decreasing the mechanical threshold induced by mechanical stimuli. As shown in Fig. 1, the mechanical threshold in the STZ-induced diabetic rats was significantly decreased after $14 \mathrm{~d}$ of STZ treatment, suggesting the establishment of mechanical hyperalgesia in diabetic rats. The decrease of paw withdrawal responses to mechanical stimuli in diabetic rats was diminished by PRF, which lasted for at least $6 \mathrm{~d}$, suggesting that PRF is capable of inhibiting mechanical hyperalgesia in diabetic rats (P<0.05, Fig. 1). PRF had no effect on mechanical sensation in normal animals ( $P>0.05$, Fig. 1 ).

\section{Effect of PRF on heat hyperalgesia}

STZ caused significant heat hyperalgesia by decreasing paw withdrawal latency to heat. As shown in Fig. 2, the paw withdrawal latency to heat kept declining after STZ-treatment. Three weeks after STZ-treatment, the paw withdrawal latency to heat in STZ group was significantly lower compared to that in the normal group ( $\mathrm{P}<0.05$ Fig. 2$)$, suggesting the establishment of heat hyperalgesia in diabetic rats. PRF had no effect on paw withdrawal latency to heat in normal animals ( $P>0.05$, Fig. 2). The decreased paw withdrawal latency to heat in STZ-treated rats was partially reversed by PRF at time points $1 \mathrm{~d}$ and $3 \mathrm{~d}$ (P<0.05, Fig. 2).

\section{Effect of PRF on cold allodynia}

Cold allodynia was induced in diabetic rats as shown in Fig. 3, and the paw withdrawal latency to cold stimuli kept declining after STZ-treatment. Three weeks after STZ-treatment, the paw withdrawal latency to cold stimuli in STZ group was significantly lower compared to that in the normal group, suggesting the establishment of cold allodynia in diabetic rats. PRF had no effect on paw withdrawal latency to cold stimuli in normal animals. The decreased paw withdrawal latency to cold stimuli in STZ-treated rats was partially reversed by PRF, which lasted for at least $6 \mathrm{~d}(\mathrm{P}<0.05$, Fig. 3). 


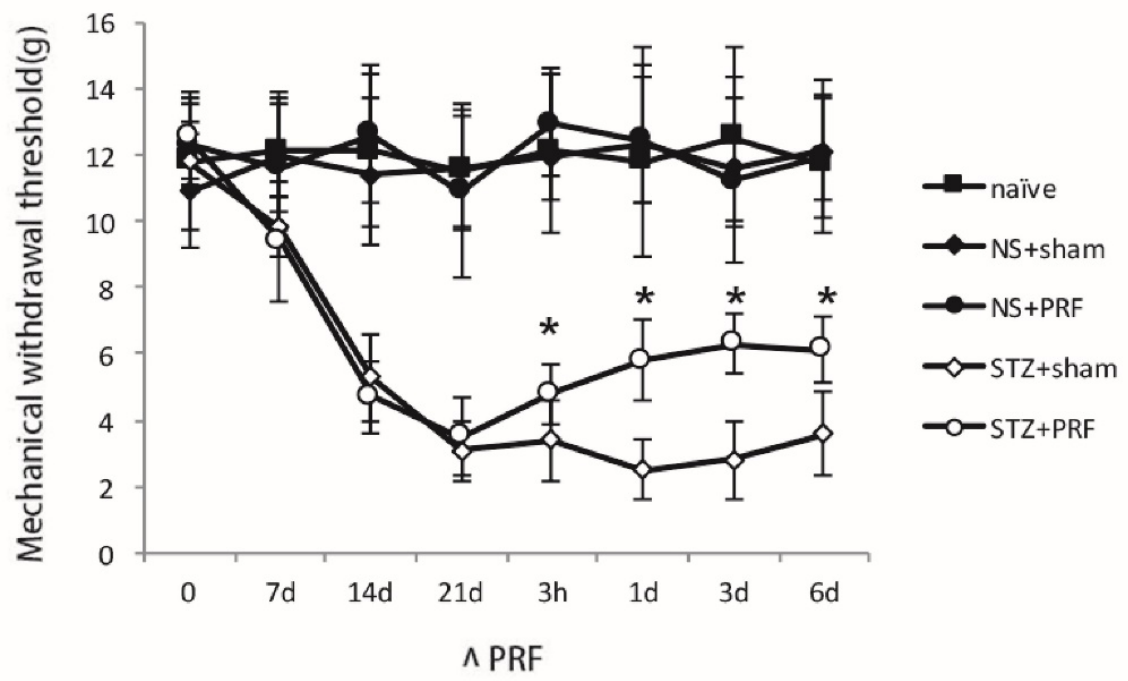

Figure 1. Effect of L5 and L6 PRF treatment on mechanical hypersensitivity in diabetic rats. The mechanical threshold in STZ-induced diabetic rats was significantly lower than that in normal rats. PRF alleviates diabetes-induced mechanical hypersensitivity $(n=10$ in each group). PRF significantly increased the mechanical threshold in diabetic rats. Data are mean $\pm S D, * p<0.05$ for the comparison to the diabetic rats treated with sham operation.

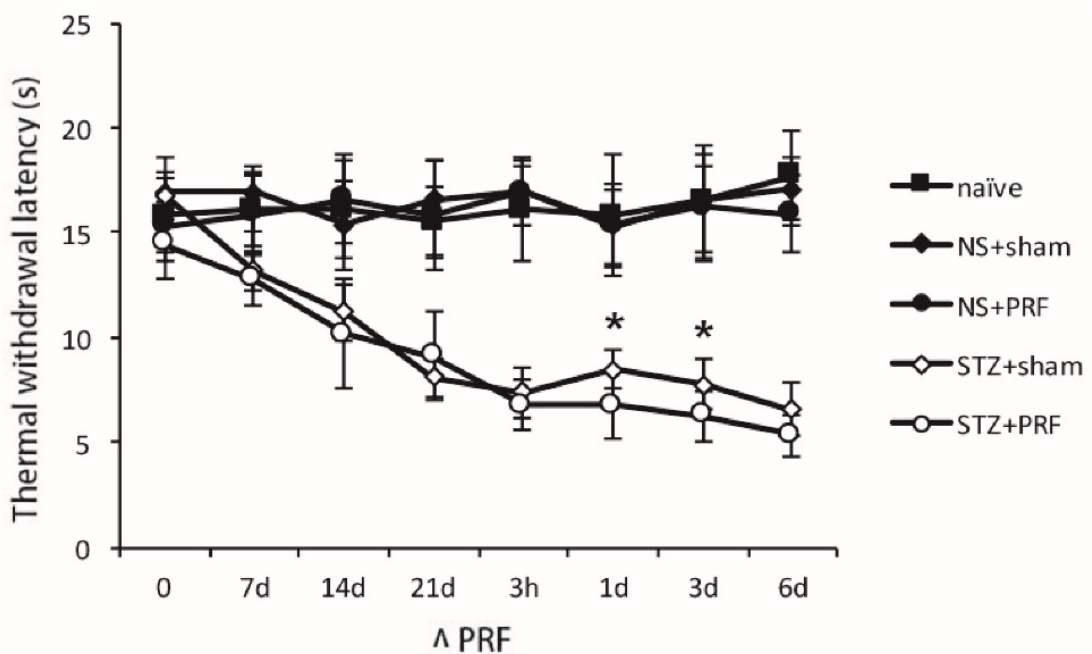

Figure 2. Effect of L5 and L6 PRF treatment on heat hyperalgesia in diabetic rats. STZ caused significant heat hyperalgesia by decreasing paw withdrawal latency to heat $(n=10$ in each group). PRF alleviates diabetes-induced heat hyperalgesia. Data are mean $\pm S D, * p<0.05$ for the comparison to the diabetic rats treated with sham operation.

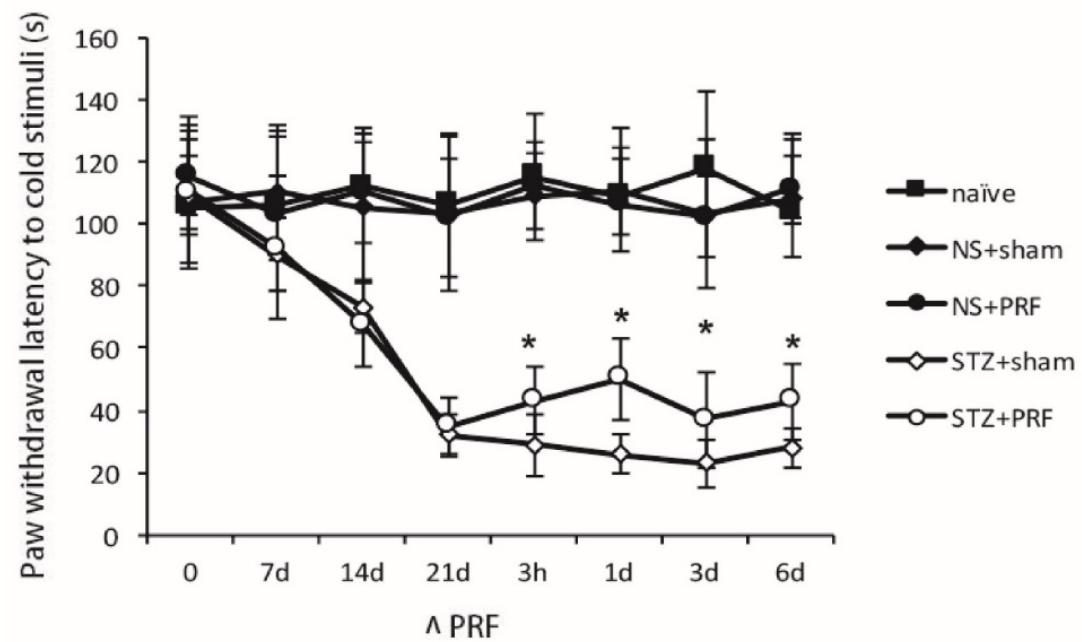

Figure 3. Effect of L5 and L6 PRF treatment on cold sensitivity in diabetic rats. STZ caused significant cold hypersensitivity by decreasing paw withdrawal latency to cold ( $n=10$ in each group). PRF alleviates diabetes-induced cold hypersensitivity. Data are mean $\pm S D, * p<0.05$ for the comparison to the diabetic rats treated with sham operation. 


\section{Effect of PRF on behaviour responses in formalin test}

The formalin-evoked flinching behaviour and spinal glutamate release were measured in conscious and freely moving rats. Flinching behaviour of the paw that received formalin injection was measured concurrently with spinal microdialysis. Control rats exhibited a bi-phasic flinching response with the active phases separated by a quiescent period of inactivity (Fig. 4). In diabetic rats, flinching was increased within minutes of the injection of formalin, and this increase was maintained throughout all three phases of the monitoring period (Fig. 4). PRF decreased formalin-evoked flinches throughout all three phases of the monitoring period after the intraplantar formalin injection (Fig. 4).

\section{Effect of PRF on formalin-evoked release of amino acids in spinal CSF dialysate}

The basal concentrations of the excitatory amino acid glutamate in spinal dialysates were significantly lower in diabetic rats than in controls $(7.8 \pm 0.8$ vs. $3.4 \pm 0.5 \mathrm{mmol} / \mathrm{l}$, NS+sham vs. STZ+sham), whereas there was no difference in the basal levels of aspartate, taurine, glycine or citrulline between the control and diabetic rats (aspartate: $1.1 \pm 0.2$ vs. $1.2 \pm 0.3$, taurine $8.6 \pm 0.8$ vs. $9.4 \pm 0.9$, glycine: $24.3 \pm 3.2$ vs. $22.3 \pm 2.9$, citrulline $6.5 \pm 0.8$ vs. $6.9 \pm 0.9 \mathrm{mmol} / \mathrm{l}, \quad P>0.05)$. Injection of $5 \%$ formalin into the hind paw of control rats evoked a significant increase in glutamate above basal levels that was most marked in the first fraction following paw stimulation (Fig 5). Transient increases of aspartate, taurine, glycine and citrulline were also noted in the fraction immediately following formalin injection. Diabetic rats also exhibited a marked increase in glutamate levels above baseline following paw formalin injection (Fig. 5). Absolute levels were significantly lower in the fractions collected during the initial $10 \mathrm{~min}$ after formalin compared to those of control rats, although the change relative to the basal levels of the same animals was of a higher magnitude to that observed in control rats (Fig. 5). PRF significantly attenuated the formalin-induced release of glutamate in diabetic rats during the initial $20 \mathrm{~min}$ after formalin compared to those of sham-treated rats (Fig. 5. STZ+PRF vs. STZ+sham). PRF also significantly suppressed the formalin-induced release of glutamate in nondiabetic rats during the initial 5 min after formalin compared to those of sham-treated rats (Fig. 5. NS+PRF vs. NS+sham). PRF had no significant effect on the formalin-induced release of aspartate, taurine, glycine and citrulline (supplemental figures in supplementary material).

\section{Discussion}

PRF was developed to reduce neuronal damage [11], making it potentially suitable for patients with DNP. The mechanisms underlying PRF-induced analgesia have not been fully elucidated. The application of PRF adjacent to the DRG is related to a short- and a long-term increase in neuronal markers in the DRG and the dorsal horn [16-18]. Electron microscopic studies demonstrated small histological changes including enlarged endoplasmic reticulum cisterns or increased cytoplasm vacuoles. Conversely, conventional radiofrequency (CRF) resulted in significant changes: mitochondrial degeneration and a loss of nuclear membrane and neurolemma integrity $[19,20]$.

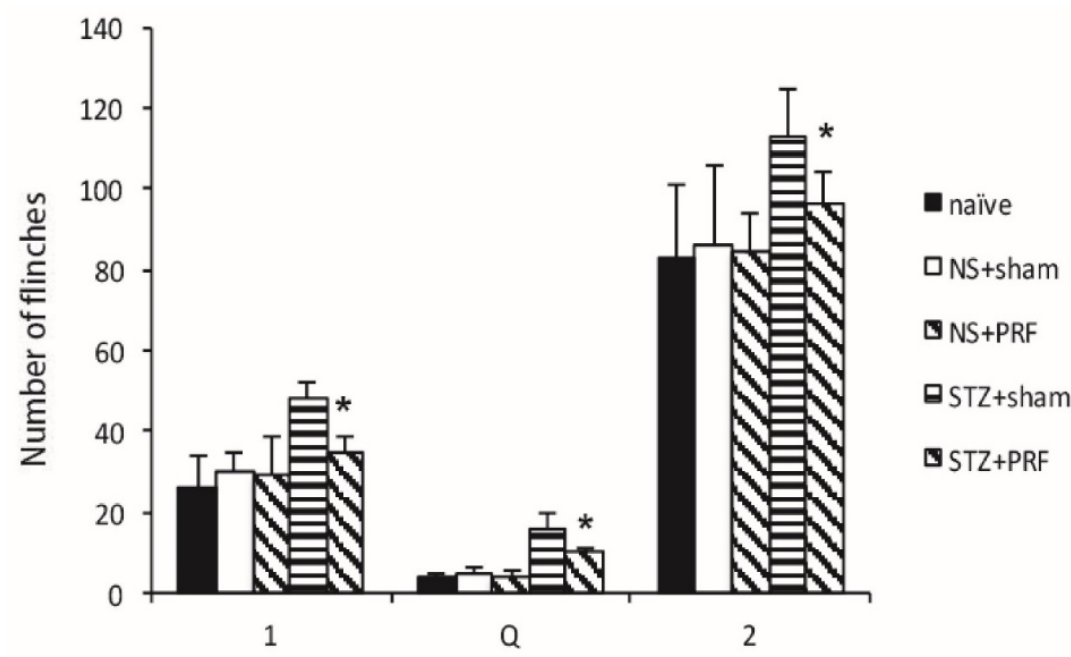

Figure 4. Effect of L5 and L6 PRF treatment on the formalin-evoked behaviour response in diabetic rats. Formalin-evoked paw flinching, presented as sum flinches counted during minutes $0-1,1-2,2-3,3-4$ and $4-5$ (phase 1 ), during minutes 5-6, 10-11 and 15-16 (phase Q) and during minutes 20-21, 25-26, 30-31, 35-36, 40-41, $45-46,50-51,55-56$ and $60-61$ (phase 2) of the formalin test in control (filled bars) and diabetic (open bars) rats. Data are mean \pm SD of $n=10$ rats per group, $*_{p}<0.05$ for the comparison to the diabetic rats treated with sham operation (STZ+sham). 


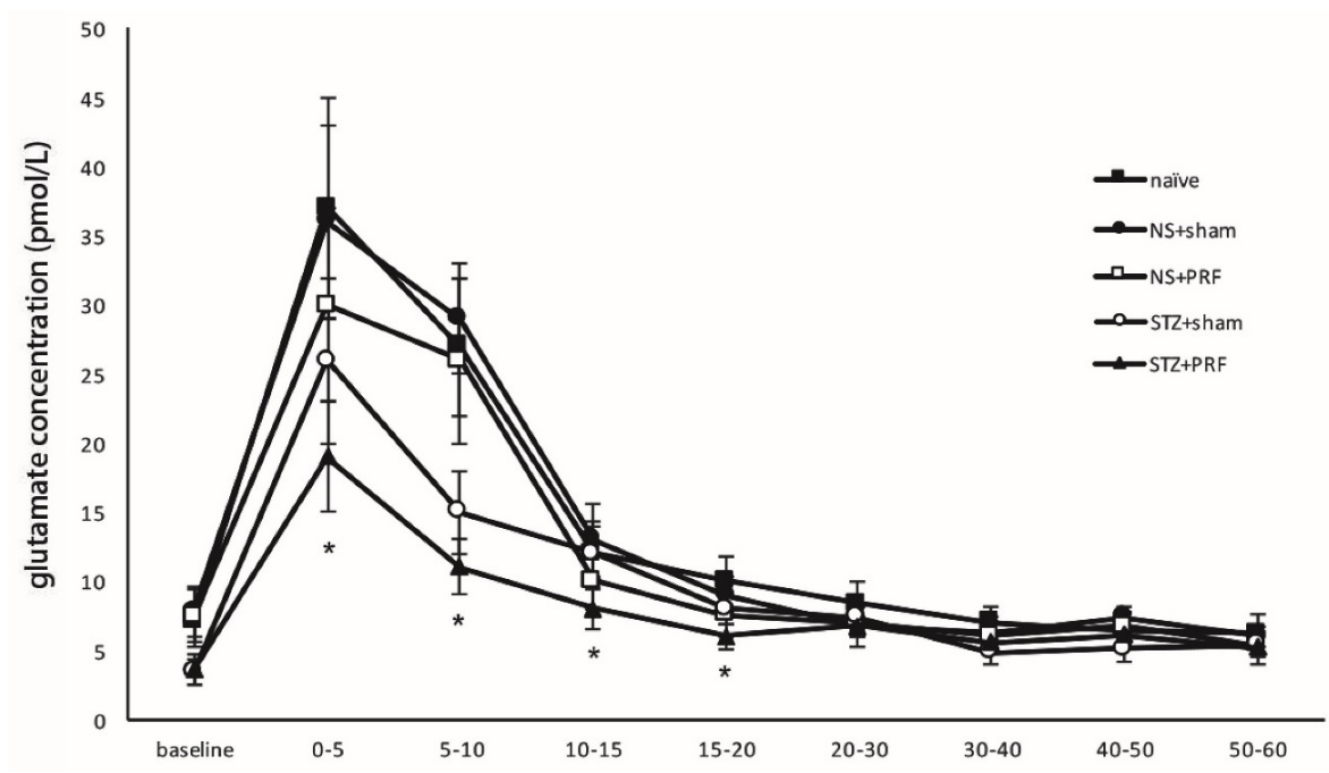

Figure 5. Effect of L5 and L6 PRF treatment on the formalin-evoked levels of amino acid in spinal dialysate in diabetic rats. Amount of glutamate in spinal CSF dialysates from control and diabetic rats prior to, and following, injection of $0.5 \%$ formalin in the hind paw. The amount of glutamate (pmol) was collected per minute within each fraction. Data are mean \pm SD of $n=10$ rats per group, ${ }^{*} p<0.05$ for the comparison to the diabetic rats treated with sham operation.

An effect was seen on myelinated nerve fibers after the application of CRF or PRF on the sciatic nerve. The application of CRF resulted in severe degeneration [21] of the distal peripheral nerve, in contrast to the PRF group, where only separation in myelin configuration were observed [21]. Nevertheless, small electron microscopic changes have also been observed after application of PRF on the sciatic nerve. The damage appears to be more pronounced for the nociceptive C-fibers and Aסfibers than the tactile $A \beta$ - fibers [22]. This might explain differential analgesic effects without greatly interfering with the tactile sensory input.

The present study investigated the potential beneficial effect of PRF on diabetes-induced hyperalgesia and allodynia in rats. We found that PRF was capable of attenuating diabetes-induced heat hyperalgesia, mechanical hyperalgesia and cold allodynia. Further studies showed that PRF was capable of decreasing formalin-induced behaviour responses and suppressing the glutamate release following formalin intraplantar injection. Interestingly, although PRF inhibited the glutamate level increase by a small amount in control rats, PRF had little effect on nociception in these animals. This finding suggests that the PRF effect is not sufficient to affect normal nociceptive transmission in the spinal dorsal horn. Similar to these results, it has been shown that although PRF can reverse hyperalgesia caused by inflammation, it has no effect on normal nociception $[13,23]$. All of the findings indicate the therapeutic potential of PRF in the treatment of diabetes-induced hyperalgesia and allodynia, without affecting the normal nociception, which might be associated with attenuating the glutamatergic neurotransmission.

The spinal dorsal horn is a critical site for the transmission and modulation of nociception. Glutamate released from the central terminals of nociceptive primary afferents is an important neurotransmitter in the spinal dorsal horn. Consistent with a previous study [24], the basal concentrations of glutamate in the CSF were lower in diabetic than in control rats. In other studies $[8,25]$, the frequency of glutamatergic spontaneous excitatory postsynaptic currents (sEPSCs) and the amplitude of evoked monosynaptic and polysynaptic EPSCs were significantly higher in diabetic rats than that in control rats. The inhibitory effects of the glutamate receptors on sEPSCs and on evoked monosynaptic and polysynaptic EPSCs were significantly greater in diabetic than in control rats. The percentage of lamina II neurons in which sEPSCs and evoked EPSCs affected by glutamate receptor inhibitors was also significantly higher in diabetic rats than that in control rats. STZ administration significantly altered the postsynaptic glutamateric neurotransmission.

The formalin test combined with intrathecal microdialysis offers the opportunity to measure the changes in pain-related behaviours and spinal neurotransmitter levels, which allows us to examine the effects of diabetes on ongoing spinal modulation of sensory processing. In our study, the percentage of glutamate change relative to the basal level after formalin intraplantar injection was much higher in diabetic than in control rats. These differences likely reflect increased glutamate release from the primary 
afferent terminals to the spinal dorsal horn neurons in diabetic rats. This enhanced glutamate release contributes to the hyperexcitability of dorsal horn neurons and the enhancement of DNP [7]. Furthermore, PRF treatment at DRG suppressed the increased glutamate levels in the CSF after formalin injection in diabetic rats. These results suggest that the analgesic action of PRF involves the inhibition of glutamate release in the nociceptive transmission in the spinal cord.

Increasing evidence also shows that glutamate receptor-dependent activation of the MAPK pathway is critical for the development of neuronal plasticity and is an important molecular mechanism for the long-lasting behavioural plasticity in various pain conditions [26, 27]. All three subtypes of ionotropic glutamate receptors and three subgroups of mGluRs seem to consistently modulate the MAPK pathway in a stimulatory fashion [28]. Active MAPKs translocate to the nucleus to activate a specific set of transcription factors for the facilitation of target gene expression [29]. This glutamate receptor-dependent, MAPK-mediated stimulus-transcription coupling controls the development of multiple forms of synaptic plasticity related to various normal and abnormal neural activities, including various pain conditions [30]. In an animal model of experimental painful diabetic neuropathy, ERK, JNK and p38 phosphorylation was significantly increased in the spinal cord and in DRG, which reflects an increase in their activation [31]. MAPK inhibitor treatment attenuated diabetic-induced mechanical hyperalgesia confirmed that MAPK played a role in the development of DNP [31,32].

The mechanisms underlying the effect of PRF treatment have not been well defined. Accumulating evidence shows that PRF may inhibit spinal sensitization by down-regulating spinal MAPK activations and reduce the synthesis of proinflammatory/pronociceptive mediators via distinct molecular and cellular mechanisms, resulting in the attenuation of persistent pain. In our previous study, PRF reduced CFA-induced mechanical hypersensitivity by attenuating JNK activation in the spinal dorsal horn [23]. In a spinal nerve ligation model, PRF significantly diminished nerve ligation-induced mechanical allodynia and thermal by downregulating p38 and ERK activation [33] and TNF production. In a spared nerve injury model, PRF can decrease mechanical and cold pain behaviours for more than $28 \mathrm{~d}$ and suppress the activation of ERK in the spinal cord [34]. All of these reports support that the analgesic mechanisms of PRF are closely associated with the suppression of MAPK activation. In diabetic rats, it is logical to infer that PRF decreased glutamatergic neurotransmission and subsequently suppressed the MAPK activation and decreased DNP.

The application of PRF in the management of diabetic neuropathic pain should be considered carefully, as the mechanisms involved in the immediate versus the delayed phase may differ. In our examination, one time PRF at $21 \mathrm{~d}$ after STZ administration provided analgesia for one week. Diabetic polyneuropathy is primarily a symmetrical sensory polyneuropathy, initially affecting the distal lower extremities. In clinical practice, for example, PRF at L4, L5 and S1 DRGs might be able to produce a long-term relief of diabetic foot pain. Furthermore, PRF relieved both the first and second phases of formalin test. Given as a pre-treatment, PRF may be acting pre-emptively to prevent central sensitization, an effect that has been shown often for NMDA antagonists [35]. Our previous report showed that PRF relieved pain or hyperalgesia in animal models of inflammatory pain such as after complete Freund's adjuvant (CFA) injection [13, 36]. As these models reflect nociception starting immediately after injection, it shows that PRF can act rapidly on pain. In clinical practice, for example, we might apply PRF before the amputation, debridement or skin grafting of the diabetic foot to attenuate postoperative persistent pain. One more PRF treatment may be applied to provide optimal results for patients.

Collectively, we provide a more logical and convincing explanation for the analgesic mechanism of PRF in DNP. The application of PRF may create an electric field at the tip of the electrode that does not induce a histological lesion but delivers brief electrical pulses or electromagnetic waves sufficient to inhibit glutamate release in the spinal cord of diabetic rats. The inhibition of glutamate release might further affect the activity of glutamate receptors, as well as expressing their downstream pain-related and MAPK expression that attenuated the pain sensitivity in the spinal dorsal horn to provide central sensitization. This central suppression effect of PRF attenuates pain hypersensitivity, as evidenced by our behavioural findings.

\section{Conclusion}

PRF is able to attenuate diabetes-induced hyperalgesia and allodynia, which might be associated with suppressing spinal glutamatergic transmission. PRF might be used as an effective treatment, especially with fewer side effects, for the management of abnormal sensation in DNP.

\section{Supplementary Material}

Supplementary figures.

http://www.medsci.org/v13p0984s1.pdf 


\section{Acknowledgements}

This work was supported, in part, by grant Nos. CMRPG8A1011, CMRPG8A1012 and CMRPG8A1013 from Chang Gung Memorial Hospital Research, Kaohsiung, Taiwan, and by grant Nos. 101-2314-B-182-090-, 102-2314-B-182-029- and 103-2314-B-182-045-MY3 from the Taiwan National Science Council Research, Taipei, Taiwan.

\section{Author contributions}

$\mathrm{CR}$ and $\mathrm{YH}$ conceived and designed the experiments; $\mathrm{SY}$ and $\mathrm{CH}$ performed the experiments; $\mathrm{CR}$ and $\mathrm{SY}$ analyzed the data; $\mathrm{CH}$ contributed reagents/materials/analysis tools; SY wrote the paper.

\section{Conflicts of Interest}

The authors declare no conflict of interest.

\section{References}

1. Ziegler D, Fonseca V. From guideline to patient: a review of recent recommendations for pharmacotherapy of painful diabetic neuropathy. Journal of diabetes and its complications. 2015; 29: 146-56.

2. Tesfaye S. Advances in the management of diabetic peripheral neuropathy. Current opinion in supportive and palliative care. 2009; 3: 136-43.

3. Tesfaye S, Selvarajah D. Advances in the epidemiology, pathogenesis and management of diabetic peripheral neuropathy. Diabetes/metabolism research and reviews. 2012; 28 Suppl 1: 8-14.

4. Kangrga I, Randic M. Outflow of endogenous aspartate and glutamate from the rat spinal dorsal horn in vitro by activation of low- and high-threshold primary afferent fibers. Modulation by mu-opioids. Brain research. 1991; 553: 347-52.

5. Sluka KA, Jordan HH, Willis WD, Westlund KN. Differential effects of $\mathrm{N}$-methyl-D-aspartate (NMDA) and non-NMDA receptor antagonists on spinal release of amino acids after development of acute arthritis in rats. Brain research. 1994; 664: 77-84

6. Calcutt NA, Chaplan SR. Spinal pharmacology of tactile allodynia in diabetic rats. British journal of pharmacology. 1997; 122: 1478-82.

7. Chen SR, Samoriski G, Pan HL. Antinociceptive effects of chronic administration of uncompetitive NMDA receptor antagonists in a rat model of diabetic neuropathic pain. Neuropharmacology. 2009; 57: 121-6.

8. Wang XL, Zhang HM, Chen SR, Pan HL. Altered synaptic input and GABAB receptor function in spinal superficial dorsal horn neurons in rats with diabetic neuropathy. The Journal of physiology. 2007; 579: 849-61.

9. Chua NH, Vissers KC, Sluijter ME. Pulsed radiofrequency treatment in interventional pain management: mechanisms and potential indications-a review. Acta neurochirurgica. 2011; 153: 763-71.

10. Van Zundert J, Patijn J, Kessels A, Lame I, van Suijlekom H, van Kleef M. Pulsed radiofrequency adjacent to the cervical dorsal root ganglion in chronic cervical radicular pain: a double blind sham controlled randomized clinical trial. Pain. 2007; 127: 173-82.

11. Shabat S, Pevsner $Y$, Folman $Y$, Gepstein R. Pulsed radiofrequency in the treatment of patients with chronic neuropathic spinal pain. Minimally invasive neurosurgery : MIN. 2006; 49: 147-9.

12. Byrd D, Mackey S. Pulsed radiofrequency for chronic pain. Current pain and headache reports. 2008; 12: 37-41.

13. Yang $\mathrm{CH}$, Chen $\mathrm{KH}$, Huang HW, Sheen-Chen SM, Lin CR. Pulsed radiofrequency treatment attenuates increases in spinal excitatory amino acid release in rats with adjuvant-induced mechanical allodynia. Neuroreport. 2013; 24: 431-6.

14. Ferrari LF, Cunha FQ, Parada CA, Ferreira SH. A novel technique to perform direct intraganglionar injections in rats. J Neurosci Methods. 2007; 159: 236-43.

15. Malmberg $\mathrm{AB}$, Yaksh TL. Spinal nitric oxide synthesis inhibition blocks NMDA-induced thermal hyperalgesia and produces antinociception in the formalin test in rats. Pain. 1993; 54: 291-300.

16. Higuchi Y, Nashold BS, Jr., Sluijter M, Cosman E, Pearlstein RD. Exposure of the dorsal root ganglion in rats to pulsed radiofrequency currents activates dorsal horn lamina I and II neurons. Neurosurgery. 2002; 50: 850-5; discussion 6.

17. Van Zundert J, de Louw AJ, Joosten EA, Kessels AG, Honig W, Dederen PJ, et al. Pulsed and continuous radiofrequency current adjacent to the cervical dorsal root ganglion of the rat induces late cellular activity in the dorsal horn. Anesthesiology. 2005; 102: 125-31.
18. Hamann W, Abou-Sherif S, Thompson S, Hall S. Pulsed radiofrequency applied to dorsal root ganglia causes a selective increase in ATF3 in small neurons. European journal of pain. 2006; 10: 171-6.

19. Erdine S, Yucel A, Cimen A, Aydin S, Sav A, Bilir A. Effects of pulsed versus conventional radiofrequency current on rabbit dorsal root ganglion morphology. European journal of pain. 2005; 9: 251-6.

20. Protasoni M, Reguzzoni M, Sangiorgi S, Reverberi C, Borsani E, Rodella LF, et al. Pulsed radiofrequency effects on the lumbar ganglion of the rat dorsal root: a morphological light and transmission electron microscopy study at acute stage. European spine journal : official publication of the European Spine Society, the European Spinal Deformity Society, and the European Section of the Cervical Spine Research Society. 2009; 18: 473-8.

21. Tun K, Cemil B, Gurcay AG, Kaptanoglu E, Sargon MF, Tekdemir I, et al. Ultrastructural evaluation of Pulsed Radiofrequency and Conventional Radiofrequency lesions in rat sciatic nerve. Surg Neurol. 2009; 72: 496-500; discussion 1.

22. Erdine S, Bilir A, Cosman ER, Cosman ER, Jr. Ultrastructural changes in axons following exposure to pulsed radiofrequency fields. Pain Pract. 2009; 9: 407-17.

23. Chen KH, Yang CH, Juang SE, Huang HW, Cheng JK, Sheen-Chen SM, et al. Pulsed radiofrequency reduced complete Freund's adjuvant-induced mechanical hyperalgesia via the spinal c-Jun N-terminal kinase pathway. Cellular and molecular neurobiology. 2014; 34: 195-203.

24. Malmberg AB, O'Connor WT, Glennon JC, Cesena R, Calcutt NA. Impaired formalin-evoked changes of spinal amino acid levels in diabetic rats. Brain research. 2006; 1115: 48-53.

25. Li JQ, Chen SR, Chen H, Cai YQ, Pan HL. Regulation of increased glutamatergic input to spinal dorsal horn neurons by mGluR5 in diabetic neuropathic pain. Journal of neurochemistry. 2010; 112: 162-72.

26. Ji RR, Gereau RWt, Malcangio M, Strichartz GR. MAP kinase and pain. Brain research reviews. 2009; 60: 135-48.

27. Kawasaki Y, Kohno T, Zhuang ZY, Brenner GJ, Wang H, Van Der Meer C, et al. Ionotropic and metabotropic receptors, protein kinase A, protein kinase $\mathrm{C}$, and Src contribute to C-fiber-induced ERK activation and cAMP response element-binding protein phosphorylation in dorsal horn neurons, leading to central sensitization. The Journal of neuroscience : the official journal of the Society for Neuroscience. 2004; 24: 8310-21.

28. Willard SS, Koochekpour S. Glutamate, glutamate receptors, and downstream signaling pathways. International journal of biological sciences. 2013; 9: 948-59.

29. Wang JQ, Fibuch EE, Mao L. Regulation of mitogen-activated protein kinases by glutamate receptors. Journal of neurochemistry. 2007; 100: 1-11.

30. Rahn EJ, Guzman-Karlsson MC, David Sweatt J. Cellular, molecular, and epigenetic mechanisms in non-associative conditioning: implications for pain and memory. Neurobiology of learning and memory. 2013; 105: 133-50.

31. Daulhac L, Mallet C, Courteix C, Etienne M, Duroux E, Privat AM, et al. Diabetes-induced mechanical hyperalgesia involves spinal mitogen-activated protein kinase activation in neurons and microglia via N-methyl-D-aspartate-dependent mechanisms. Molecular pharmacology. 2006; 70: 1246-54.

32. Purves T, Middlemas A, Agthong S, Jude EB, Boulton AJ, Fernyhough P, et al. A role for mitogen-activated protein kinases in the etiology of diabetic neuropathy. FASEB journal : official publication of the Federation of American Societies for Experimental Biology. 2001; 15: 2508-14.

33. Lin WT, Chang CH, Cheng CY, Chen MC, Wen YR, Lin CT, et al. Effects of low amplitude pulsed radiofrequency stimulation with different waveform in rats for neuropathic pain. Conference proceedings : Annual International Conference of the IEEE Engineering in Medicine and Biology Society IEEE Engineering in Medicine and Biology Society Annual Conference. 2013; 2013: 3590-3.

34. Yeh CC, Sun HL, Huang CJ, Wong CS, Cherng CH, Huh BK, et al. Long-Term Anti-Allodynic Effect of Immediate Pulsed Radiofrequency Modulation through Down-Regulation of Insulin-Like Growth Factor 2 in a Neuropathic Pain Model. Int J Mol Sci. 2015; 16: 27156-70.

35. Coderre TJ, Van Empel I. The utility of excitatory amino acid (EAA) antagonists as analgesic agents. II. Assessment of the antinociceptive activity of combinations of competitive and non-competitive NMDA antagonists with agents acting at allosteric-glycine and polyamine receptor sites. Pain. 1994; 59: 353-9.

36. Hagiwara S, Iwasaka H, Takeshima N, Noguchi T. Mechanisms of analgesic action of pulsed radiofrequency on adjuvant-induced pain in the rat: roles of descending adrenergic and serotonergic systems. European journal of pain. 2009; 13: 249-52. 